\title{
着床前小鼠胚胎和子宫蛋白质的 生化分析及鉴定*
}

\author{
曹永清 郑 明 \\ (中国科学院动物研究所内分速室及生殖生物学开故研究实验室, 北京 100080) \\ 陈廷汉 B. Lofts \\ (香港大学动物学系)
}

关链词着床、蛋白质、等电聚焦、双向电泳、荧光自显影

哺乳动物胚胎与子官间的相互协调作用, 是着床和维持妊娠的一个重要因素. 巳知妊娠 信号 (signa1) 具有两方面作用, 对母体引起一系列的生理改变, 并使胚胎和子宫局部引起同 步的相互作用. 例如着床前羊胚胎合成的信号物质, 有延长母体的黄体功能, 并使子宫内膜蜕 膜化; 而啮齿动物子宫生成的某些因子能使胚胎发生滞育和延缓着床现象 ${ }^{[1,22}$. 本研究对着休 前发育不同阶段小鼠阫胎及其相应时期子宫组织和子宫腔冲洗液中蛋白质进行了分析和鉴 定, 其目的是为了解在着床过程中胚胎和母体子宫合成及分爫的蛋白质在质量方面的变化和 特性.

\section{一、材料和方法}

1. 动物和还胎的收集 用小鼠杂交种 $\mathrm{C} 57 \times \mathrm{A}_{2} \mathrm{G}$ 的第一代动物, 年:龄为 4-.5 周堆性 鼠经注射 PMSG 5IU/只, 44 h 后注射 hCG 5IU/只, 注射后立即交配, 次曰上午 9:00 检查阴 栓, $24 \mathrm{~h}$ 后解剖动物, 从输卵管冲出 2-细胞胚胎, 用 $50 \mu 1 \mathrm{M} 276$ 培养液 ${ }^{[3]}$ 并加盖石蜡液体外 培养 $\left(5 \% \mathrm{CO}_{2}, 95 \%\right.$ 空气, $\left.37^{\circ} \mathrm{C}\right)$, 第 2 天收集桑椹胚, 第 3 天收集胚泡, 以作分析用.

2. 子官组织和子宫液的收集分别将妊娠第 1 、2、3 天动物, 损伤颈椎后进行解剖, 用双 蒸水 $\mathrm{DDH}_{2} \mathrm{O}$ 冲洗子宫腔并收集冲洗液, 与此同时收集子宫作分析用.

3. ${ }^{3} \mathrm{H}$ - 亮虫酸挎人蛋白质合成试验取发育不同时期胚胎加人 PB 276 培养液 ${ }^{[3]}$ 和 ${ }^{3} \mathrm{H}-$ 熟氨酸 $(50 \mu \mathrm{Ci} / \mathrm{ml}) 37^{\circ} \mathrm{C}$ 餒有 $4 \mathrm{~h}$, 收集肧胎用 $\mathrm{DDH}_{2} \mathrm{O}$ 洗 3 次, 保存在 $-70^{\circ} \mathrm{C}$ 冰箱, 以待 电泳和苂光自显影分析用 ${ }^{[4]}$.

4. 等电聚焦电泳和双向电泳 用 LKB 2117 多用途电泳仪进行实验, 操作见 LKB 操 作说明书昭.

\section{二、结果和讨论}

1. 子宫组织和子宫冲洗液的蛋白质分析妊娠不同时期小鼠子宫组织等电聚焦电泳 ( $\mathrm{pH} 3-10)$ 分析结果表明, 有 3 种妊娠特异蛋白质, 它们的等电点 (PI) 分别为 5.05、5.70和 6.20 , 在非孕动物子宫组织未发现有此 3 种蛋白质 (图 1). 在 $\mathrm{pH} 4-6$ 的等电聚焦电泳图谱 本文 1990 年 9 月 18 日收到.

* 本研究工作得到香港球橧基金会资助.

第 15 期 


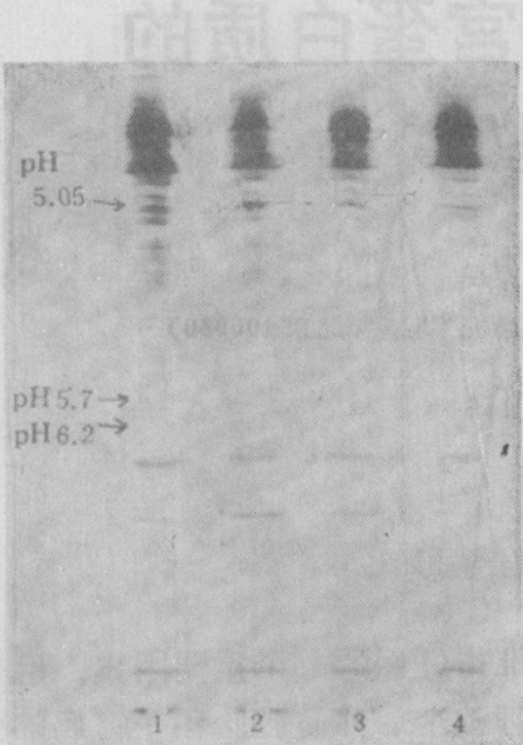

图 1 小鼠子宫组织 $\mathrm{pH}_{3}-10$ 等电聚焦图谱 （卡马氏蓝染色）

1. 2-细胞期；2.桑椹期；3. 目泡期；4.非朶期

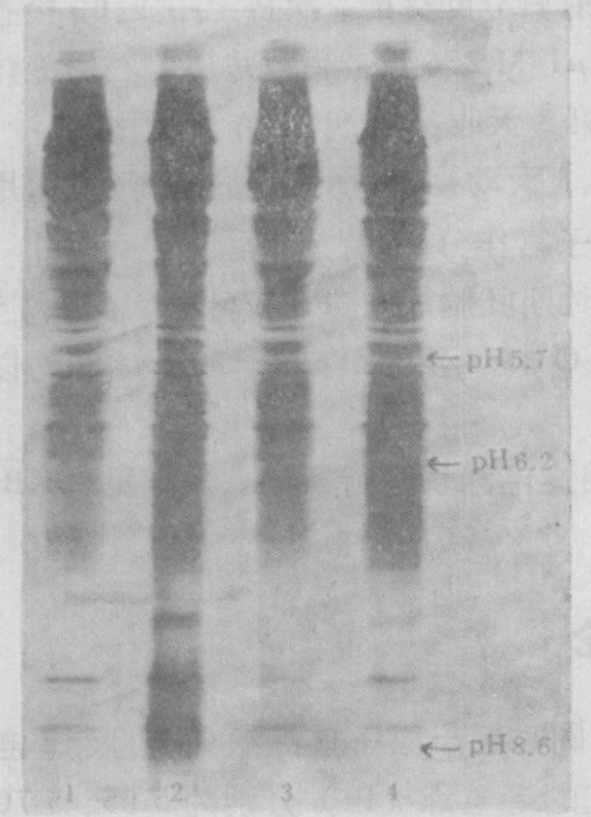

图 3 小鼠子宫冲洗液 $\mathrm{pH}_{3}-10$ 等电聚焦图谱 （卡马氏蓝和银染色）

\section{1.非㭆动物; \\ 2. 2-细胞期；3.桑楛期； 4. 胚泡期}

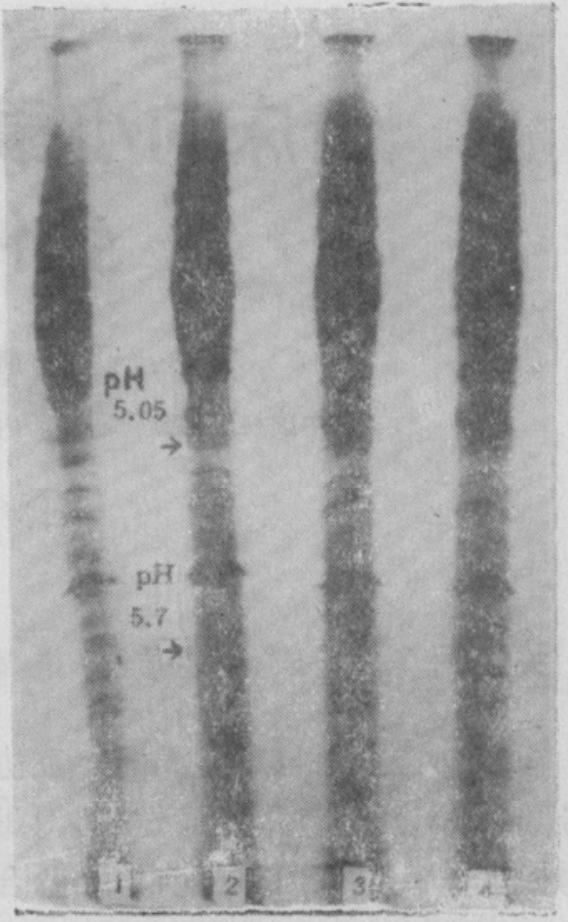

图 2 小鼠子官组织 $\mathrm{pH} 4.6$ 等电聚焦图谱 （银染色）

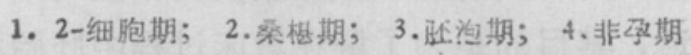

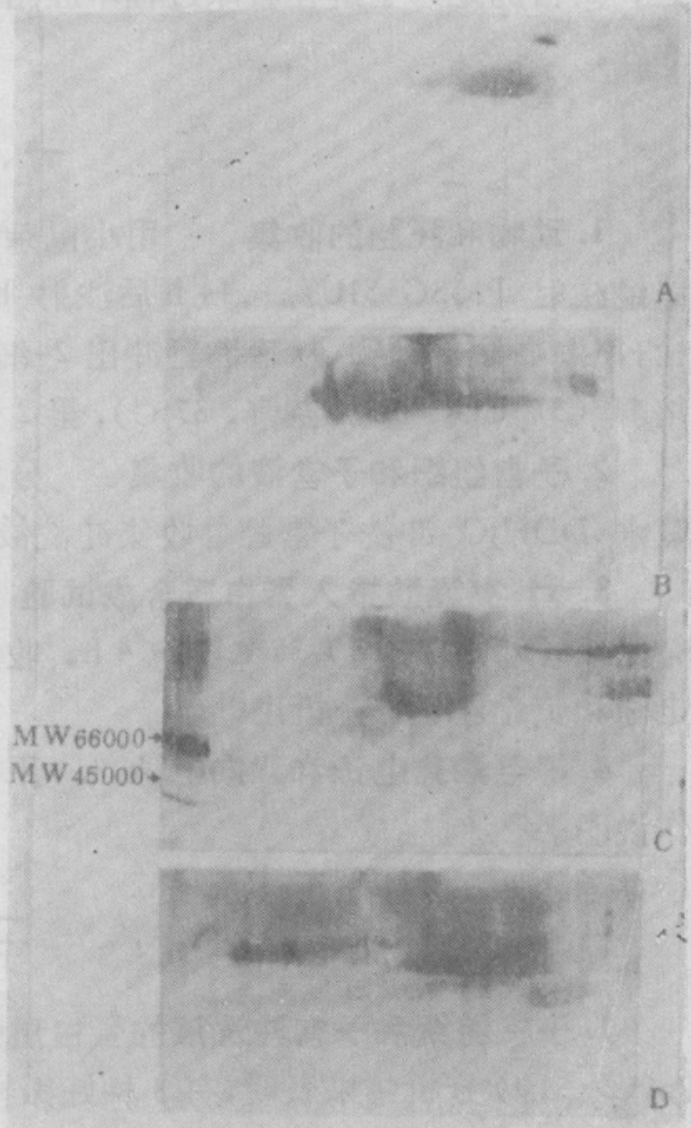

图 4 小鼠子宫冲洗液双向电泳图谱 等电乑焦 $\mathrm{pH}$ 为 4-6.
A. 非孕动物； B. 2-细胞期;
C. 桑椹期;
D. 还泡期

1991 年 
(图 2) 同样发现有 PI 为 5.05 和 5.70 的蛋白质条带.

在妊䆣不同天数小鼠子官冲洗液的 $\mathrm{pH}$ 3-10 等电聚集电泳图谱（图 3)也存在 PI 为 5.05 和 5.70 的蛋白质条带, 而非孕动物子官冲洗液未发现有此 2 种蛋白质. 从双向电泳图谱 （图 4) 可知该 2 种蛋白质的分子量范围为 66000 至 80000 . 由此可知 PI 为 5.05 和 5.70 的 2 种蛋白质是胚胎发育过程中由子宫组织合成并分泌的.

2. 音床过程中发育不同时期泼胎蛋白质的分析 实验结果发现在 $\mathrm{pH}$ 为 3-10 的等 电聚焦图谱(图 5) 中, 显示胚泡有一特异蛋白质条带, 其 PI 为 5.50, 在 2-细胞和桑椹胚的电 泳图谱中未能显示此蛋白质条带. 此外, 在桑椹胚和胚泡电泳图谱中均有一PI 为 9.0 的蛋白 质条带, 但在 2-细胞胚未能显示. 从荧光自显影图谱(图 6), 可知 ${ }^{3} \mathrm{H}$-亮氨酸主要掺人酸性蛋 白质中, 这说明在着床过程中的胚胎, 特别是桑椹胚和胚泡有较大量糖蛋白的合成.

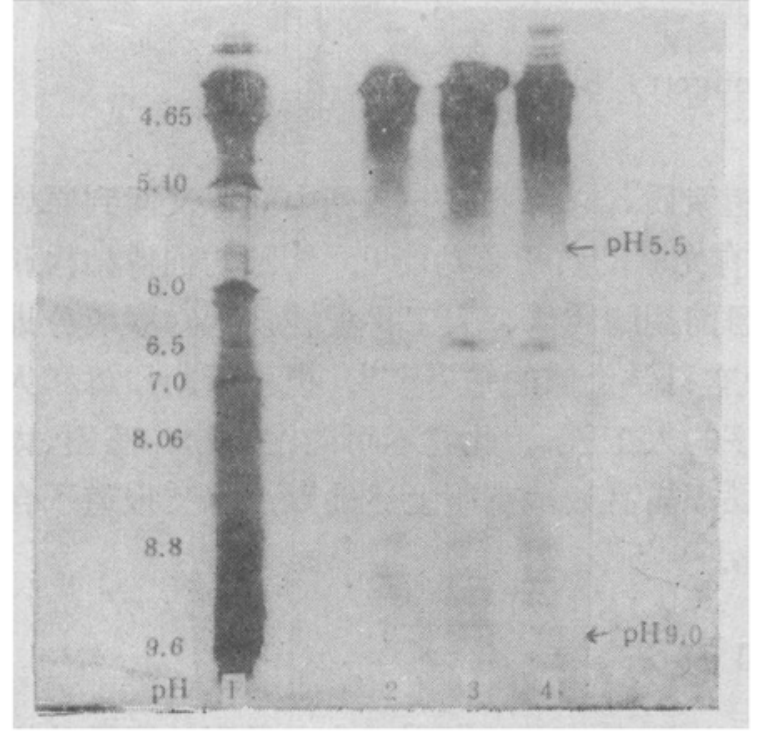

图 5 小圂胚胎 $\mathrm{pH} 3-10$ 等电聚焦图谱 1.Bio-Rad IEF 标准; 2. 2-细胞；3. 桑墈继；4.胚淘

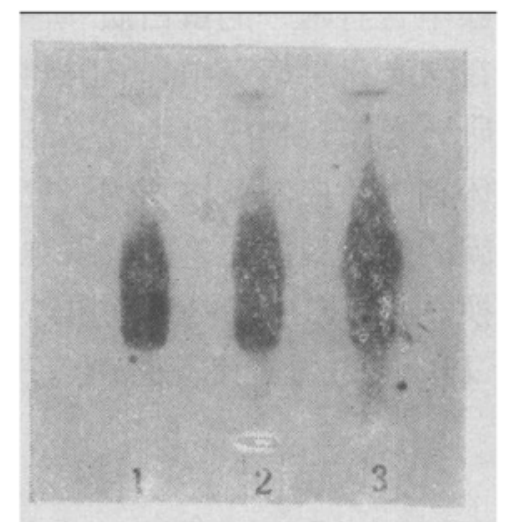

图 6 小鼠胚胎 $\mathrm{pH}_{4}$-6 等电聚焦电泳荧光自显影图谱 1. 2-细胞；2.桑椹胚：3. 胚泡

人们都认为着休过程中胚胎与子宫的各自生理活动, 彼此起着相互影响的作用. 但是, 其 作用的性质、时间和范围仍不清楚. 本研究结果表明, 着床过程中小鼠胚胎蛋白质的合成有着 质和量的变化, 在桑椹胚和胚泡有大量酸性蛋白质的合成. 这一结果与 Godkin 及其同事们 的报道相附合 ${ }^{[6]}$. 在桑椹胚和胚泡有 PI 为 9.0 的蛋白质合成, 而在 2-细胞胚胎中不显示此 种蛋白质. 此外, 肧泡能合成 PI 为 5.5 的特异蛋白质, 在 2-细胞和襄胚中均不显示此种蛋白 质. 在母体子宫方面, 无论在子宫组织和子宫冲洗液中的蛋白质, 同样也有质和量的变化, 有 2 种特异蛋白质只存在妊娠子宫组织和子官液中, 在非孕动物组织中未能发现. 根据上述实 验结果, 有理由推测, 着床过程中胚胎和子宫组织合成蛋白质的变化与分必, 可能是着床所必 需的并与完成着床有密切的关系.

\section{參考文献}

[1] Heap, R. B. et al., Br. Med. Bull., 35(1979), 129-135.

[2] Kennedy, T. G., Aust. J. Biol., 36(1983), 531-543.

[3] Yu, H. S. \& Chan, S. T. H., Pharm. Toxicol., 60(1987), 129-134.

[4] Schrader, W. T. et al., Laboratory Manual for Hormone Action and Molecular Endocrinology, 1982.

[5] LKB, Instruction sheet $1818-\mathrm{P}$ for $0.5 \mathrm{~mm}$ thin layer polyacryamide gel for electrofocusing, 1981.

[6] Godkin, J. D. et al., Endocrinology, 114(1984), 120. 